\title{
Biomechanical Differences in the Sprint Start Between Faster and Slower High-Level Sprinters
}

\author{
by \\ Milan Čoh ${ }^{1}$, Stanislav Peharec², Petar Bačić2 , Krzyszfof Mackala ${ }^{3}$
}

\begin{abstract}
The purpose of this study was to examine the kinematic and kinetic differences of the sprint start and first two steps between faster and slower high-level sprinters. Twelve male sprinters were dichotomized according to personal best 60- and 100-m times. Each participant performed five starts under constant conditions. An eight-camera system was used for 3-D kinematic analysis. Dynamic forces at the start were determined with starting blocks mounted on bipedal force plates. Measures of front and rear block total force, front and rear block maximal force, time to front and rear block peak force, total force impulse, total horizontal and vertical impulse, front and rear block force impulse, time of block clearance, block leaving velocity and block leaving acceleration were collected. Between-group comparisons were made using independent samples $t$ tests $(p<0.05)$ and by calculating effect sizes (Cohen's $d)$. Spearman's correlation coefficients were used to examine the relationships between sprint start kinematics, kinetic measures and sprint performance. Significant between-group differences were observed in rear block total force $(p=0.0059)$, rear block maximal vertical force $(p=0.0037)$ and total force impulse $(p=0.0493)$. Only front block total force significantly correlated with $100 \mathrm{~m}$ sprint performance in both the slower and faster groups $(r=0.94$ and 0.54 , respectively; $p=$ 0.05). Our findings suggest that faster sprinters show enhanced sprint start motor performance with greater force development than slower sprinters.
\end{abstract}

Key words: sprinters, block start, biomechanics, kinematics, dynamics.

\section{Introduction}

A well-executed sprint start, where the sprinter must rapidly accelerate from a stationary set position, is one of the determining factors of high performance in sprinting (Atwater, 1982; Bowman, 1975; Mackala et al., 2010). According to the literature, an effective sprint start predominantly depends on start block positioning and the body centre of gravity (BCG) in the set position (Coppenolle et al., 1990; Korchemny, 1992; Schot and Knutzen, 1992), block clearance time and force impulse on the front and rear starting blocks as well as take-off velocity and acceleration (Fortier et al., 2010; Guissard and Hainaut, 1992; Harland and Steele, 1997; Mero et al., 2006). Among these variables, Tellez and Doolittle (1984) documented that take-off acceleration accounted for $64 \%$ of the total time in a $100 \mathrm{~m}$ sprint. However, determining the optimal relationship between the body position and initial acceleration in the first two to five steps represents a specific biomechanical paradigm, where a sprinter has to integrate temporal and spatial acyclic movements into a cyclic action (Harald and Steel, 1997; Mackala et al., 2010).

Atwater (1982) analyzed several variations of the set position among different performance levels of sprinters and found no single, optimum solution appropriate for all sprinters. However,

1 - University of Ljubljana, Faculty of Sport, Ljubljana, Slovenia.

2 - Polyclinic for Physical Medicine and Rehabilitation "PEHAREC", Pula, Croatia.

3 - University of School of Physical Education, Department of Track and Field, Wroclaw, Poland. 
the literature has recommended that the most ideal position involves the hips raised above the shoulders, shoulders directly above the start line and body weight distributed on the front foot, with the leading knee forming an angle of $90-110^{\circ}$ and the rear knee $120-135^{\circ}$ (Hay, 1993). Subsequent investigations of start performance have concentrated not only on discrete body positioning and associated start block settings, but also on the development of angular velocity and maximal force by the sprinter during the start (Coh et al., 2007; Harland and Steele, 1997; Mero et al., 1992). The latter aspect is particularly important as Mero et al. (1983) found that the horizontal and vertical force exerted on the front and rear starting blocks strongly correlated $(\mathrm{r}=$ 0.74 ) with block velocity.

While research on the block start in sprinting has investigated a number of biomechanical variables (Ferro et al., 2001; Mero et al., 1992) and their interdependencies with specific motor abilities, energy processes, anthropometric characteristics and central processes of motor regulation (Gutierrez-Davila, 2006; Locatelli and Arsac, 1995; Mero et al., 2006; Prampero et al., 2005), little has been reported on inter-individual variability across different performance levels. Despite repeatedly performing a predetermined set position (in line with the aforementioned recommendations), variation in distinct kinetics and kinematics associated with the take-off can biomechanically differentiate the starting ability of sprinters.

Therefore, the purpose of the study was to determine the differences in the kinematic and dynamic characteristics quantifying the sprint start between faster (elite / international level) and slower (sub-elite / national level) sprinters. Analysis of force-time data could determine if force production during the start had a significant impact on velocity kinematics during block clearance and thus, differentiate faster sprinters from slower ones. Based on earlier biomechanical research concerning the sprint block start (Harland and Steele, 1997; Mero at al., 1983; Schot and Knutzen, 1992), it was hypothesized that start kinetics (e.g. force production) would show greater differences between sprinters than start kinematics (e.g. time of block clearance). Further insight into this issue may provide a greater understanding of block start technique and knowledge on how the block clearance phase can lead to mechanically-enhanced sprint start performance.

\section{Material and Methods}

\section{Participants}

Twelve high-level Slovenian male sprinters were recruited (age: $22.4 \pm 3.4$ years; body height: $177.6 \pm 6.9 \mathrm{~cm}$; body mass: $74.9 \pm 5.2$ $\mathrm{kg}$ ). The inclusion criterion required at least 6 years of training experience in the 60,100 or 200 $\mathrm{m}$ sprint events. According to the aims of the study, the "faster" group included members of the Slovenian national team competing at the international level $(n=6)$ and the "slower" group consisted of sprinters from regional clubs competing at the national level $(n=6)$. Differences between the groups were confirmed by comparing mean personal best times in official 60 m (faster: $6.87 \pm 0.13 \mathrm{~s}$; slower: $6.98 \pm 0.05 \mathrm{~s}$ ) and $100 \mathrm{~m}$ (faster: $10.66 \pm 0.18 \mathrm{~s}$; slower: $11.00 \pm 0.06 \mathrm{~s}$ ) runs. All participants were informed about the purpose of the study and its procedures. Written consent was obtained and the athletes, as voluntary participants, could withdraw at any time of the research. The study was approved by the Human Ethics Committee of the University School of Physical Education in Wroclaw, Poland, and conducted in accordance with the Declaration of Helsinki.

\section{Procedures}

Testing was conducted at the beginning of May, before the competitive season, several months after the participants' individual sprint training regime began. This time period was selected as it ensured that the participants would be physically fit and present correct starting technique. The sprinters were tested during a quasi-standard sprint training session in a biomechanical laboratory. The participants were asked to maintain their normal intake of food and fluids, but to avoid any physical activity 24 hours and food 3 hours before testing. The testing protocol required each participant to perform five maximal-effort $4 \mathrm{~m}$ block start sprints interspersed with $5 \mathrm{~min}$ rest. Test-retest reliability of the block start position was assessed by interclass correlation coefficients calculated from three consecutive block starts. High reliability was found $(r=0.93)$, which indicated that the applied 
protocol (block start plus $4 \mathrm{~m}$ run-out) was uniform across the sprinters. A standardized 20 min warm-up consisting of jogging, stretching exercises, skipping drills and short accelerations was performed before the sprints. Sprinters were allowed to individually position the starting blocks. It was found that all participants utilized a bullet-type start where the distance between the front foot toe and rear foot toe was between 25 and $30 \mathrm{~cm}$ in the on your marks position (Hay, 1993). The starting pistol was directly connected to a photocell (AMES) timing system (Microgate, Bolzano, Italy) to measure reaction time in order to determine if maximum effort was applied. Each start was performed according to international athletic rules.

\section{Measurements}

Standing body height was measured with the head positioned in the Frankfurt plane using a fixed wall-mounted stadiometer (Seca Z05-PF321, Liverpool, England) and recorded to the nearest $0.1 \mathrm{~cm}$. Body mass was measured on a calibrated digital scale (Seca 862, Liverpool, England) with accuracy of $0.1 \mathrm{~kg}$. All anthropometric measurements were taken twice by a trained nurse in the morning 1 day before testing in a biomechanical laboratory at the Peharec Polyclinic for Physiotherapy and Rehabilitation in Pula, Croatia. Two independent force plates (Kistler Type 9286A, Winterthur, Switzerland) operating at $800 \mathrm{~Hz}$ were placed on an indoor gym surface, on which two starting block pads were installed (Figure 1). The development of force in the horizontal/vertical direction $\left(\mathrm{F}_{\mathrm{xy}}\right)$, vertical direction $\left(\mathrm{F}_{\mathrm{y}}\right)$ and horizontal anterior-posterior direction ( $\left.\mathrm{F}_{\mathrm{x}}\right)$ was recorded (Figures 2, 3). Measures selected for analysis were as follows: front and rear block total force $(\mathrm{N})$, front and rear block maximal force in both the horizontal and vertical directions $(\mathrm{N})$, time to front and rear block peak force $(\mathrm{ms})$, total force impulse $(\mathrm{N} \cdot \mathrm{s})$, total horizontal and vertical impulse $(\mathrm{N} \cdot \mathrm{s})$ and front and rear block force impulse $(\mathrm{N} \cdot \mathrm{s})$. Start kinematics were assessed by an eight CCD camera system (SMART-e 600, BTS Bioengineering, Padua, Italy) recording at $200 \mathrm{~Hz}$ and $768 \times 576$ pixel resolution. A segment coordinate system (SCS) was defined based on 16 retroreflective markers placed on the head, shoulders, upper arm, lower arm, trunk, thigh, shank and foot of each participant (Figure 1). SMART Analyser software was used to digitize the anatomical markers in 3-D in the set position, at the start of the take-off, and during the first two steps. Discrete kinematic measures included reaction time (ms), total block reaction time (ms), front and rear block reaction times $(\mathrm{ms})$, block velocity $\left(\mathrm{m} \cdot \mathrm{s}^{-}\right.$ $\left.{ }^{1}\right)$, block acceleration $\left(\mathrm{m} \cdot \mathrm{s}^{-2}\right)$ and the length $(\mathrm{cm})$ and contact time (ms) of the first and second step out of the block.

\section{Statistical analysis}

Descriptive statistics (mean $\pm S D$ ) were calculated for all variables. The Shapiro-Wilk test indicated a normal distribution for all variables. Comparisons between the faster and slower sprinters were examined by unpaired Student's $t$ tests and by calculating the effect size using Cohen's $d$ (Thalheimer and Cook, 2002). Effect sizes were interpreted as negligible $(d \geq 0.2)$, small $(0.2 \leq d \leq 0.5)$, medium $(0.5 \leq d \leq 0.8)$ or large $(0.8 \leq$ d). Spearman's correlation coefficients were used to examine the relationships between the kinematic and kinetic variables of the sprint block start and sprint performance. Spearman's correlation coefficients were interpreted as small (0.1 - 0.3), medium (0.3 - 0.5) and large (0.5 - 1.0) (Mukaka, 2012). The level of significance was set at $p \leq 0.05$ or $p \leq 0.01$. Data processing was performed with SPSS for Windows 15.0 (Chicago, IL, USA).

\section{Results}

Table 1 provides the anthropometric and sprint block start performance data for the faster and slower sprinters. Age, body height and mass were similar between the groups. When we compared the personal best $100 \mathrm{~m}$ times, the difference was statistically significant between the faster and slower group $(p<0.05)$.

Few differences were observed among the kinematic measures between the faster and slower sprinters (Table 2). Significant between-group differences were found for block velocity $(d=1.57$, $p=0.0294)$ and rear block reaction time $(d=1.29, p$ $=0.0493$ ). Differences between the groups for front block reaction time $(d=1.11)$ and total reaction time $(d=0.85)$ were also observed, although they were not significant. No differences in the block distance from the starting line were found, confirming that the sprinters adopted a similar position.

Conversely, six measures of force during 
the block start were significantly $(p<0.05)$ different between the faster and slower sprinters (Table 3). These included rear block total force, rear block maximal vertical force and total force impulse. No differences in front and rear maximal horizontal forces and front block maximal vertical force were observed. There was a significant difference in total horizontal and vertical force impulse and it was of greater magnitude in the faster sprinters than slower ones.

Spearman's correlations between sprint performance, kinematics and kinetic variables are presented in Table 4 . This analysis revealed that only front block total force was strongly associated with $100 \mathrm{~m}$ sprint performance and this was observed in both the slower and faster groups ( $r=0.94$ and 0.54 , respectively).

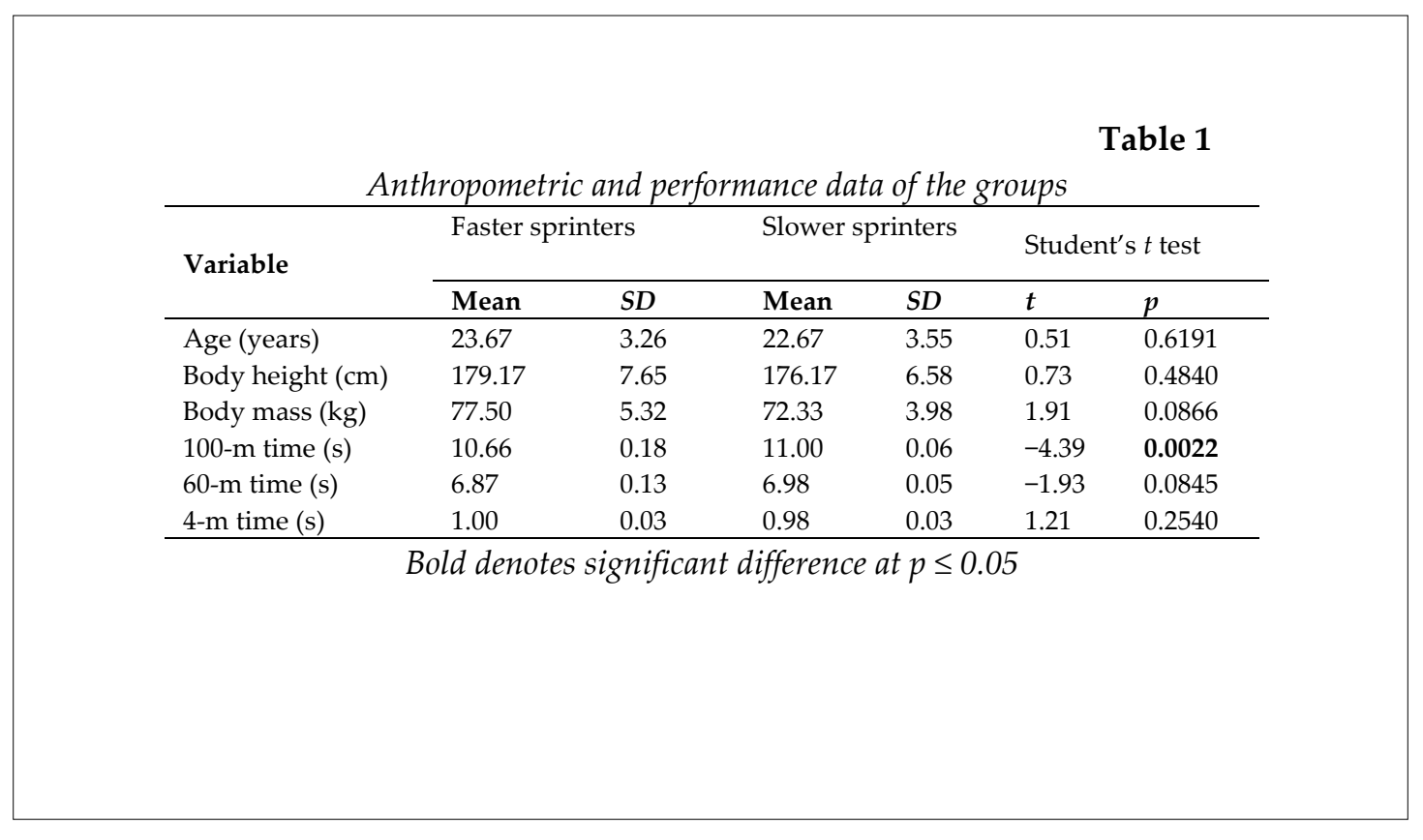

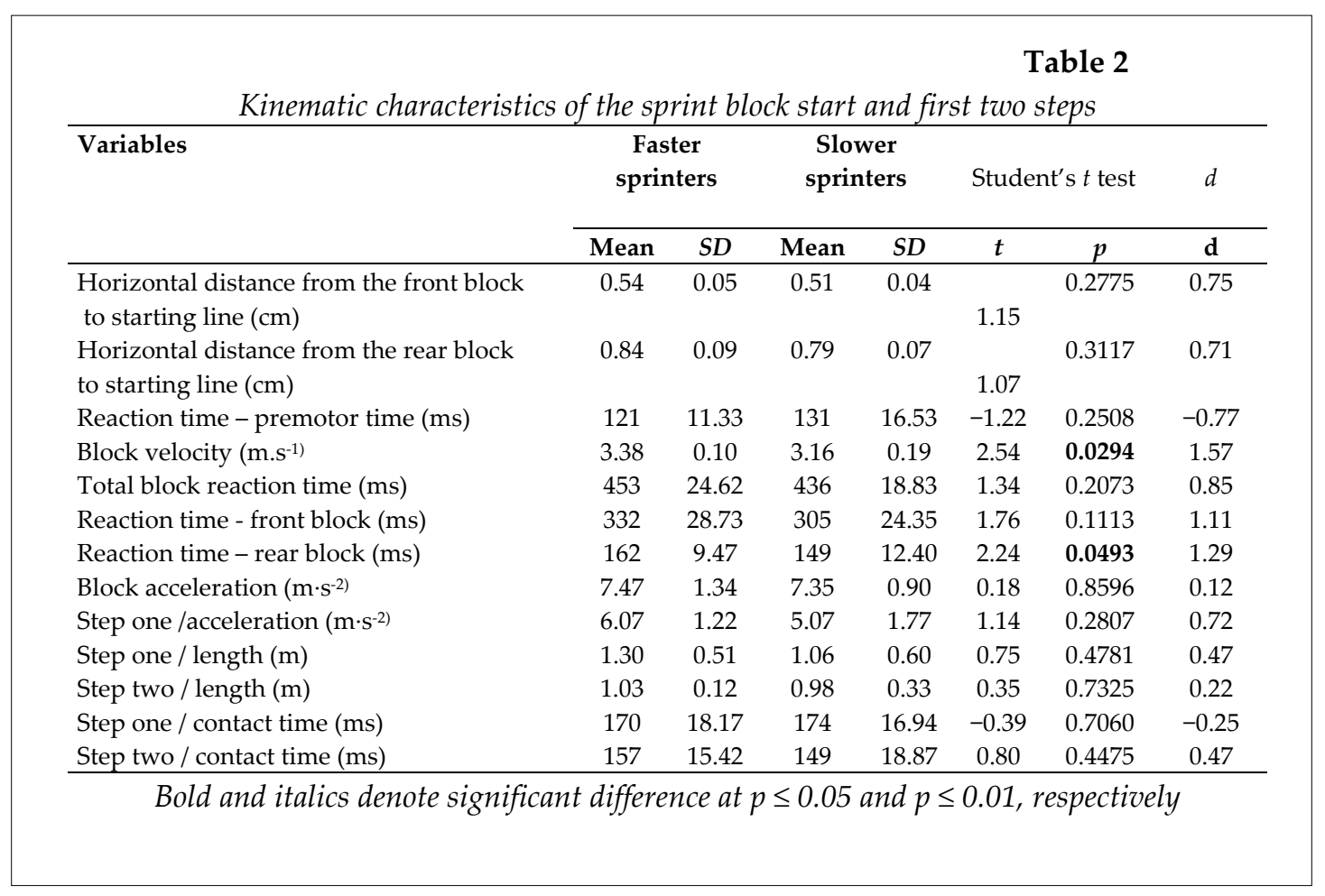


Table 3

Kinetic characteristics of sprint block start

\begin{tabular}{|c|c|c|c|c|c|c|c|}
\hline \multirow[t]{2}{*}{ Variables } & \multicolumn{2}{|c|}{ Faster sprinters } & \multicolumn{2}{|c|}{ Slower sprinters } & \multicolumn{2}{|c|}{ Student's t test } & \multirow[b]{2}{*}{$d$} \\
\hline & Mean & $S D$ & Mean & $S D$ & $t$ & $p$ & \\
\hline Total force / front block (N) & 1104 & 82.53 & 1073 & 56.21 & 0.76 & 0.4781 & 0.48 \\
\hline Total force / rear block $(\mathrm{N})$ & 913 & 89.23 & 771 & 55.09 & 3.32 & 0.0059 & 2.10 \\
\hline $\begin{array}{l}\text { Maximal force / front block } \\
\text { horizontal }(\mathrm{N})\end{array}$ & 461 & 51.05 & 398 & 56.73 & 2.02 & 0.0669 & 1.28 \\
\hline $\begin{array}{l}\text { Maximal force / rear block } \\
\text { horizontal }(\mathrm{N})\end{array}$ & 460 & 58.12 & 423 & 45.50 & 1.23 & 0.2453 & 0.78 \\
\hline $\begin{array}{l}\text { Maximal force / front block } \\
\text { vertical }(\mathrm{N})\end{array}$ & 1019 & 69.99 & 978 & 43.12 & 1.22 & 0.2508 & 0.80 \\
\hline $\begin{array}{l}\text { Maximal force / rear block } \\
\text { vertical }(\mathrm{N})\end{array}$ & 795 & 91.29 & 645 & 41.55 & 3.66 & 0.0037 & 2.32 \\
\hline $\begin{array}{l}\text { Time to peak force / front block } \\
\text { (ms) }\end{array}$ & 262 & 22.11 & 242 & 15.22 & 1.83 & 0.0924 & 1.15 \\
\hline $\begin{array}{l}\text { Time to peak force / rear block } \\
\text { (ms) }\end{array}$ & 72 & 12.81 & 70 & 8.06 & 0.32 & 0.7560 & 0.20 \\
\hline $\begin{array}{l}\text { Force impulse of front block } \\
(\mathrm{N} \cdot \mathrm{s})\end{array}$ & 221.3 & 15.8 & 178.3 & 13.1 & 5.13 & 0.0004 & 3.24 \\
\hline $\begin{array}{l}\text { Force impulse of rear block } \\
(\mathrm{N} \cdot \mathrm{s})\end{array}$ & 76.7 & 8.8 & 71.1 & 6.7 & 1.24 & 0.2435 & 0.78 \\
\hline Force impulse - total $(\mathrm{N} \cdot \mathrm{s})$ & 294.3 & 21.1 & 269.5 & 17.9 & 2.20 & 0.0493 & 1.39 \\
\hline Horizontal impulse - total $(\mathrm{N} \cdot \mathrm{s})$ & 140.7 & 11.5 & 112.8 & 10.4 & 4.41 & 0.0018 & 2.79 \\
\hline Vertical impulse - total $(\mathrm{N} \cdot \mathrm{s})$ & 256.1 & 9.7 & 209.8 & 8.9 & 8.62 & 0.0001 & 5.45 \\
\hline
\end{tabular}

Bold and italics denote significant difference at $p \leq 0.05$ and $p \leq 0.01$, respectively

Table 4

Spearman correlation between the kinematics and kinetic ariables of sprint block start and sprint performance

\begin{tabular}{llllllll}
\hline \multirow{2}{*}{ Variable } & \multicolumn{3}{l}{ Faster sprinters } & & & & \\
\cline { 2 - 8 } & [1] & [2] & [3] & [4] & [5] & [6] & [7] \\
\hline [1] 100 m & - & 0.77 & -0.49 & -0.77 & 0.14 & $\mathbf{0 . 5 4}$ & 0.31 \\
[2] 4 m & 0.77 & - & -0.03 & -0.60 & 0.26 & -0.26 & 0.49 \\
[3] Block velocity & -0.49 & -0.03 & - & 0.43 & 0.54 & 0.31 & 0.03 \\
[4] Velocity/acceleration & -0.77 & -0.60 & 0.43 & - & -0.26 & 0.66 & -0.09 \\
[5] Block acceleration & 0.14 & 0.26 & 0.54 & -0.26 & - & 0.14 & 0.49 \\
[6] Total force / front block & $\mathbf{0 . 5 4}$ & 0.26 & 0.31 & 0.66 & 0.14 & - & 0.60 \\
[7]Total force / rear block & 0.31 & 0.49 & 0.03 & -0.09 & 0.49 & 0.60 & - \\
\hline
\end{tabular}

Bold denotes significant difference at $p \leq 0.05$

\begin{tabular}{llllllll}
\hline \multirow{2}{*}{ Variable } & \multicolumn{3}{l}{ Slower sprinters } & & & \\
\cline { 2 - 8 } & [1] & [2] & [3] & [4] & [5] & [6] & [7] \\
\hline [1] 100 m & - & 0.14 & 0.03 & -0.37 & -0.43 & $-\mathbf{0 . 9 4}$ & 0.66 \\
[2] $4 \mathrm{~m}$ & 0.14 & - & 0.47 & 0.06 & -0.46 & -0.29 & -0.12 \\
[3] Block velocity & 0.03 & 0.47 & - & 0.58 & 0.41 & 0.06 & 0.17 \\
[4] Velocity/acceleration & -0.37 & 0.06 & 0.58 & - & 0.77 & 0.60 & -0.37 \\
[5] Block acceleration & -0.43 & -0.46 & 0.41 & 0.77 & - & 0.66 & -0.26 \\
[6] Total force / front block & $\mathbf{0 . 9 4}$ & -0.29 & 0.06 & 0.60 & 0.66 & - & -0.60 \\
[7]Total force / rear block & 0.66 & -0.12 & 0.17 & -0.37 & -0.26 & -0.60 & - \\
\hline
\end{tabular}

Bold denotes significant difference at $p \leq 0.05$ 

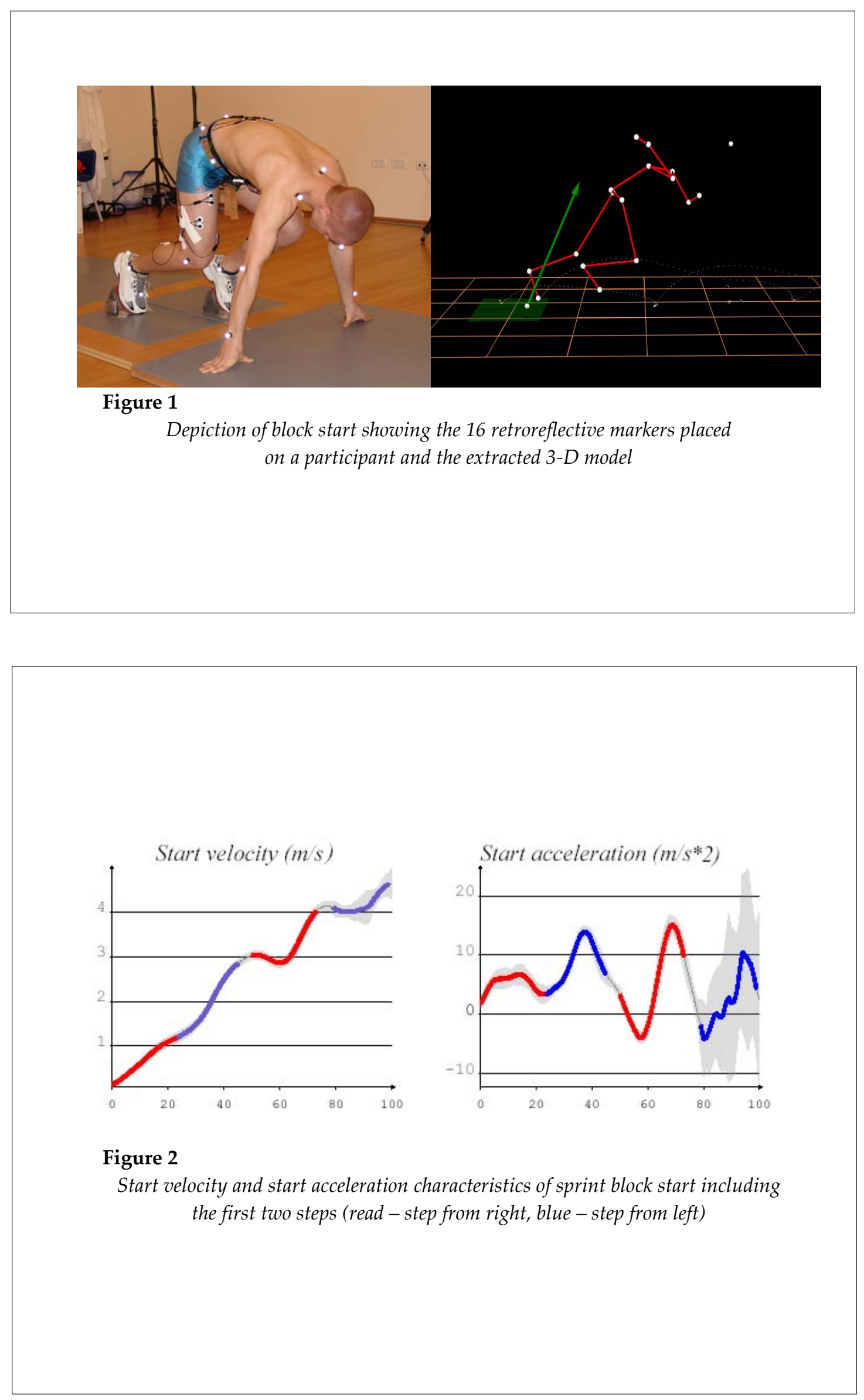

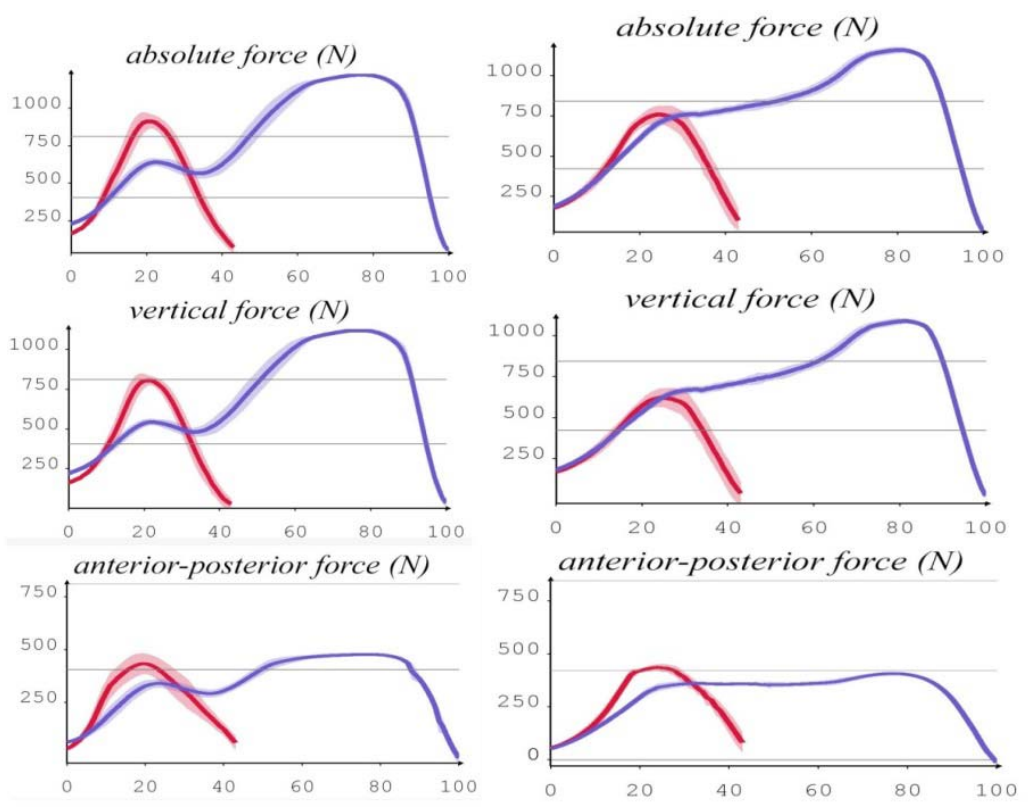

\section{Figure 3}

Front and rear sprint block start force characteristics (total force development in the vertical and horizontal directions) (read - right leg double push-of, blue - left leg double push-of)

\section{Discussion}

We identified a number of kinematic and kinetic variables associated with start block performance that differentiated faster sprinters from slower ones. However, no between-group differences were found in the $4 \mathrm{~m}$ sprint executed after the block start, which may have been due the relatively short running distance. Other studies have also reported a lack of differences in run times of 4-5 m sprints (Coh et al., 1998; Fortier et al., 2005). In spite of the small sample size, between-group differences were pronounced due to the large effect sizes for most of the selected variables $(\mathrm{d} \geq 0.8)$. The faster sprinters exhibited a reduced total force difference between the front and rear block compared with the slower sprinters $(17.3 \%$ vs. $28.2 \%$, respectively). Statistically significant differences between the faster and slower sprinters were also observed with regard to rear block total force $(913 \mathrm{~N}$ vs. $771 \mathrm{~N}, \mathrm{p}=0.0059$, respectively) and rear block vertical maximal force $(795 \mathrm{~N}$ vs. $645 \mathrm{~N}, \mathrm{p}=$ 0.0037 , respectively). It is likely that the greater force generated by the rear foot allowed the faster sprinters to achieve significantly greater take-off velocity compared to the slower sprinters (3.38 $\mathrm{m} \bullet \mathrm{s}-1$ vs. $3.16 \mathrm{~m} \bullet \mathrm{s}-1 ; \mathrm{p}=0.0294$ ).

These results suggest that faster sprinters can better optimize force distribution as well as produce more force on both starting blocks. This finding confirms Harland and Steele's (1997) and Fortier's (2005) reports where faster sprinters displayed higher rear maximum force than slower athletes. This finding is also congruent with the front block peak forces registered by other researchers (Guissard and Duchateau, 1990; 
Harland et al., 1995; McClements et al., 1996; Natta and Brenier, 1998) and supports the observation that performance in sprinting demands a high rate of force application (Coh et al., 2007; Harald and Steel 1997; Mero et al., 1993).

The generation of high force underlies other important factors of block start performance such as minimizing block clearance time as well as increasing block velocity and acceleration (Fortier et al., 2005; Harald and Steel, 1997). However, our correlation analysis did not support this assumption. During the block clearance phase, the faster sprinters were found to produce significantly greater total impulse $(294.3 \pm 21.1$ $\mathrm{N} \bullet \mathrm{s} \neg)$ than their slower counterparts $(269.5 \pm 17.9$ $\mathrm{N} \bullet \mathrm{s} \neg$ ). As force impulse is determined by (1) duration of the applied force (which did not differentiate between the two groups), (2) force magnitude (with differences registered in rear block total force and vertical rear block maximal force) and (3) the rate of force development (greater in faster sprinters), it can be assumed that the higher values of the latter two variables corresponded to the ability of faster sprinters to leave the blocks during the push phase at a higher velocity (Slawinski et al., 2010).

Several authors have reported starting block velocity to vary between 3.40 and $3.90 \mathrm{~m} \bullet \mathrm{s}-$ 1 (Coh, 2008; Harisson and Comyns, 2006; Hunter et al., 2004; Ozsu, 2014). As previously mentioned, block velocity in the group of faster sprinters was $3.38 \pm 0.10 \mathrm{~m} \bullet \mathrm{s}-1$, while in the slower group block it equalled $3.16 \pm 0.19 \mathrm{~m} \bullet \mathrm{s}-1 \quad(\mathrm{p}=0.0294)$. Although this was not measured in the present study, the difference between the faster and slower sprinters may also be related to positioning of the centre of mass (CM) closer to the starting line during the push phase (Harald and Steel, 1997; Slawinski et al., 2010). By reducing $\mathrm{CM}$ displacement (keeping the $\mathrm{CM}$ as close as possible to the starting line), a sprinter is able to generate a greater start velocity and faster block clearance (Slawinski et al., 2010), which was the case in our faster sprinters. The literature has found that while elite sprinters present a horizontal $\mathrm{CM}$ between 16 and $23 \mathrm{~cm}$ from the starting line, lower-level sprinters apply distances between 25 and $28 \mathrm{~cm}$ (Bauman, 1976; Harald and Steel, 1997; Slawinski et al., 2010). Future research examining sprint start performance should include this variable to clarify its influence on start velocity.

Nonetheless, according to Hay (1993), the main difference in the set position among sprinters lies in the longitudinal distance between the front foot and rear foot, in which three modalities are commonly used: the bunch or bullet $(25-30 \mathrm{~cm})$, a medium $(30-55 \mathrm{~cm})$ or elongated $(60-70 \mathrm{~cm})$ start. There was a negligible difference in block spacing between the faster (30 $\mathrm{cm})$ and slower sprinters $(28 \mathrm{~cm})$, classifying both groups as using the bunch start. From a biomechanical perspective, this position has been described the least efficient as lower force is exerted on the starting blocks at a consequently reduced block velocity (Harald and Steel, 1997). This view was confirmed by our correlation analysis, where kinematic variables, block velocity and block acceleration were not significantly associated with the best $60 \mathrm{~m}$ and 100 $\mathrm{m}$ sprint times of the participants. No correlations with the aforementioned variables were also observed in regard to the $4 \mathrm{~m}$ sprint performed during the experiment, but this is likely due to the minimal running distance as previously mentioned. However, this does not imply that this start technique is either fundamentally incorrect or inefficient. The usage of this technique is more a matter of personal preference or due to stronger familiarization with the associated block setting. The bunch start has some benefits for a sprinter, as it allows for faster block clearance due to a decrease in contact time with the starting blocks (Henry, 1952).

It is clearly documented within the literature that ground contact time is an important predictor of sprinting performance (Hunter et al., 2004; Mero et al., 1992, 2006; Slawinski et al., 2010), where times between 160 and $184 \mathrm{~ms}$ were reported in elite athletes (Hunter et al., 2004; Mero and Komi, 1990; Mero et al., 1992). Interestingly, we recorded contact times of $170 \pm 18.2 \mathrm{~ms}$ (first step) and $157 \pm 15.4 \mathrm{~ms}$ (second step) in the faster sprinters and $174 \pm 16.94 \mathrm{~ms}$ (first step) and $149 \pm$ $18.87 \mathrm{~ms}$ (second step) in the slower sprinters. The lack of significant differences may suggest that both groups of sprinters were able to generate similar force in the first two steps of initial acceleration. This may also indicate that both groups shared a similar skill set in transferring acyclic body movements (block clearance technique) into a cyclic movement (first steps of 
acceleration). Furthermore, this may be a consequence of a short contact time with the block during start, which was continued in the following steps. However, we noted a relatively large albeit non-significant difference in the length of the first step $(24 \mathrm{~cm})$ in favour of the faster sprinters. In turn, the second step was of almost identical length as a difference of only 5 $\mathrm{cm}$ was observed. Moreover, when considering the 4-m sprint times (involving two additional steps), nearly equivalent times were achieved by the both groups (faster sprinters $=1.0 \mathrm{~s}$ and slower sprinters $=0.98 \mathrm{~s}$ ).

The study has certain limitations that should be acknowledged. A larger sample size would have afforded greater statistical power. Additionally, the inclusion of sprinters of a higher performance level (particularly in regard to personal best $100 \mathrm{~m}$ times) would have allowed a better differentiation between "faster" and "slower" sprinters in our analysis. Measurement of lower extremity strength could have also bolstered our analysis, as muscle strength is associated with the rate of force development during the block start. Future research should include strength testing as it may better elucidate the relationships between block start kinematics and kinetics and thus, aid in identifying the variables that characterize elite sprint start performance.

\section{Conclusion}

The present study found that faster sprinters showed significantly enhanced block start performance in some kinematic and kinetic variables compared to slower sprinters. Our findings suggest that faster sprinters show motor patterns of greater force development (rear block total force, rear block vertical maximal force and the rate of force development) than their slower counterparts despite the collective employment of the bullet-type start. While the ability to generate force should underlie other important indicators of block start performance such as block clearance time, block velocity and block acceleration, this was not confirmed in our correlation analysis. From a practical standpoint, the results of this study should encourage coaches and sprinters to search for a more effective block start position such as by modifying current block settings.

\section{Acknowledgements}

The authors have no professional relationships with companies or manufacturers that might benefit from this study. The results do not constitute endorsement of any product. We would like to thank a dedicated group of sprinters for their outstanding performance in the present study.

\section{References}

Atwater A. Kinematic analyses of sprinting. Track and Field Quarterly Review, 1982; 82: 12-16

Baumann W. Kinematic and dynamic characteristics of the sprint start. In P.V. Komi (eds.), Biomechanics VB. University Park Press, Baltimore, 1975; 194-199

Coppenolle H, Delecluse C, Goris M, Diels R, Kraayenhof, H. An evaluation of the starting action of world class female sprinters. Track Tech., 1990; 90: 3581-3582

Čoh M. Biomechanical diagnostic methods in athletic training. Faculty of Sport. University of Ljubljana; 2008

Čoh M, Peharec S, Bačić P. The sprint start: Biomechanical analysis of kinematic, dynamic and electromyographic parameters. New Stud Athl., 2007; 22(3): 29-38

Coh M, Jost B, Skof B, Tamazin K, Dolenec A. Kinematic and kinetic parameters of the sprint start and start acceleration model of top sprinters. Gymnica, 1998; 28: 33-42

Ferro A, Rivera A, Pagola I. Biomechanical analysis of the $7^{\text {th }}$ World Championship in Athletics Seville 1999. New Stud Athl., 2001; 16(1-2): 25-60

Fortier S, Basset F, Mbourou A, Faverial J, Teasdale N. Starting block performance in sprinters: a statistical method for identifying discriminant parameters of performance and an analysis of the effect of providing feedback over a 6 - week period. J Sports Sci Med., 2010; 4: 134-143 
Guissard N, Duchateau J. Electromyography of the sprint start. J Hum Mov Stud, 1990; 18: 97-106

Guissard N, Hainaut K. EMG and mechanical changes during sprint start at different front block obliquities. Med Sci Sports Exerc., 1992; 24(11): 1257-1263

Gutierrez-Davila M, Dapena J, Campos J. The Effect Muscular Pre-Tensing on the Sprint Start. J Appl Biomech., 2006; 22: 194-201

Harland MJ, Andrews MH, Steele, JR. Instrumented start blocks: A quantitative coaching aid. In: XIII International Symposium for Biomechanics in Sport. Ed: Bauer T. Ontario, 367- 370; 1995

Harland M, Steele J. Biomechanics of the Sprint Start. Sports Med., 1997; 23(1): 11-20

Harrison D, Comyns T. Biomechanics of the Sprint Start. Available at: http://cis.squirming.net/category/athletics/219/. Accessed on 26.07.2006

Hay JG. The biomechanics of sport technique. $4^{\text {th }}$ ed. PrenticeHall; 1993

Henry FM. Force-time characteristics of sprint start. Res Q., 1952; 23(10): 301-318

Hunter J, Marshall R, McNair P. Interaction of Step Length and Step Rate during Sprint Running. Med Sci Sports Exerc., 2004; 36(2): 261-271

Korchemny R. A new concept for sprint start and acceleration training. New Stud Athl., 1992; 7(4): 65-72

Locatelli E, Arsac L. The mechanics and energetics of the 100m sprint. New Stud Athl., 1995; 10(1): 81-87

McClements J, Sanders L, Gander B. Kinetic and kinematic factors related to sprint starting as measured by Saskatchewan Sprint Start Team. New Stud Athl., 1996; 11(2-3): 133-135

Mero A, Komi P. Reaction time and electromyographic activity during a sprint start. Eur J Appl Physiol., 1990; 61: 73-80

Mero A, Luhtanen P, Komi P. A biomechanical study of the sprint start. Scand J Sports Sci., 1983; 5(1): 20-28

Mero A, Komi P, Gregor R. Biomechanics of Sprint Running. Sport Med., 1992; 13(6): 376-392

Mero A, Kuitunen S, Harland M, Kyrolainen H, Komi P. Effects of muscle - tendon length on joint moment and power during sprint starts. J Sports Sci., 2006; 24(2): 165-173

Mukaka MM. A guide to appropriate use of Correlation coefficient in medical research. Malawi Med J., 2012; 24(3): 69-71

Natta F, Breniere Y. Influence of the initial posture on the dynamics of sprint start from starting blocks. Science et Motricité, 1998; 34: 44-51

Ozsu I. Biomechanical structure of sprint start and effect of biological feedback methods on sprint start performance. Turk J Sport Exe., 2014; 16(1): 72-79

Prampero P, Fusi S, Morin B, Antonutto C. Sprint running: a new energetic approach. J Exp Biol., 2005; 208: 2809-2816

Schot P, Knutzen K. A Biomechanical Analysis of Four Sprint Start Positions. Res. Q. Exerc. Sport., 1992; 63(2): 137-147

Slawinski J, Bonnefoy A, Leveque M, Ontanon G, Cheze L. Kinematic and Kinetic Comparison of Elite and Well-Trained Sprinters During Sprint Start. J Strength Cond Res., 2010; 24(4): 896-905

Tellez T, Doolittle D. Sprinting from start to finish. Track Tech., 1984; 88: 2802-2805

Thalheimer W, Cook S. How to calculate effect sizes from published research articles: A simplified methodology. Available at: http://work learning.com/effect_sizes.htm. Accessed on 11.01.2016

\section{Corresponding author:}

\section{Krzysztof Mackala}

University School of Physical Education in Wroclaw (AWF Wrocław),

Department of Track and Field,Ul. Paderewskiego 35, 51-612 Wrocław, Poland

Phone: +48 71347 3140; Fax: +48 71347 3149; Mobile phone: +48 605272433

Email: krzysztof.mackala@awf.wroc.pl 